\title{
Transcatheter Mitral Valve Repair in Patients with Heart Failure: A Meta-Analysis
}

\author{
Ajay Vallakati Ayesha K Hasan Konstantinos Dean Boudoulas \\ Division of Cardiovascular Diseases, The Ohio State University Wexner Medical Center, Columbus, OH, USA
}

\section{Keywords}

Transcatheter mitral valve repair · Secondary mitral regurgitation · Heart failure

\begin{abstract}
Background: Severe secondary mitral regurgitation (MR) is associated with poor prognosis in heart failure patients with left ventricular systolic dysfunction. Few observational and randomized controlled studies demonstrated the efficacy of transcatheter mitral valve repair in heart failure patients with significant MR. A meta-analysis of published studies was performed to evaluate the role of transcatheter mitral valve repair using the MitraClip device in heart failure patients with significant secondary MR. Methods: A literature search was performed using PubMed, Cochran CENTRAL, and Embase databases using the search terms "percutaneous mitral valve repair" or "transcatheter mitral valve repair" and "heart failure." Studies that compared medical therapy plus transcatheter mitral valve repair using MitraClip to medical therapy alone in heart failure patients with significant secondary MR were included for pooled analysis. A random-effects model with the Mantel-Haenszel method was used to analyze the data. Results: Four studies, 2 randomized controlled and 2
\end{abstract}

nonrandomized studies met the criteria for analysis. Pooled analysis included a total of 1,421 patients, of which 746 patients underwent transcatheter mitral valve repair and 675 patients received medical therapy alone. When compared to medical therapy, transcatheter mitral valve repair significantly decreased all-cause mortality (OR $0.58,95 \% \mathrm{Cl} 0.37-$ $0.91 ; p=0.02)$. A trend toward significant reduction in rehospitalizations (OR $0.35,95 \% \mathrm{Cl} 0.12-1.00 ; p=0.05$ ) was also observed. Periprocedural complications ranged from 7.5 to $12.6 \%$. Conclusion: Evidence from pooled analysis suggests that transcatheter mitral valve repair using MitraClip on top of medical therapy, in appropriately selected symptomatic heart failure patients with significant secondary MR, provides survival benefit and may decrease hospitalizations when compared with guideline-directed medical therapy alone.

(c) 2020 S. Karger AG, Basel

\section{Introduction}

Secondary (i.e., functional) mitral regurgitation (MR) in patients with systolic heart failure and left ventricular (LV) dilatation is due to papillary muscle displacement

\footnotetext{
Ajay Vallakati

Division of Cardiovascular Medicine, The Ohio State University

410 W 10th Avenue

Columbus, OH 43210 (USA)

ajay.vallakati@osumc.edu
} 
Fig. 1. Schematic depicting factors contributing to the development of secondary MR in ischemic and nonischemic cardiomyopathy (from ref. [1]). MR, mitral regurgitation; LV, left ventricular.
Mechanisms of mitral regurgitation in cardiomyopathy

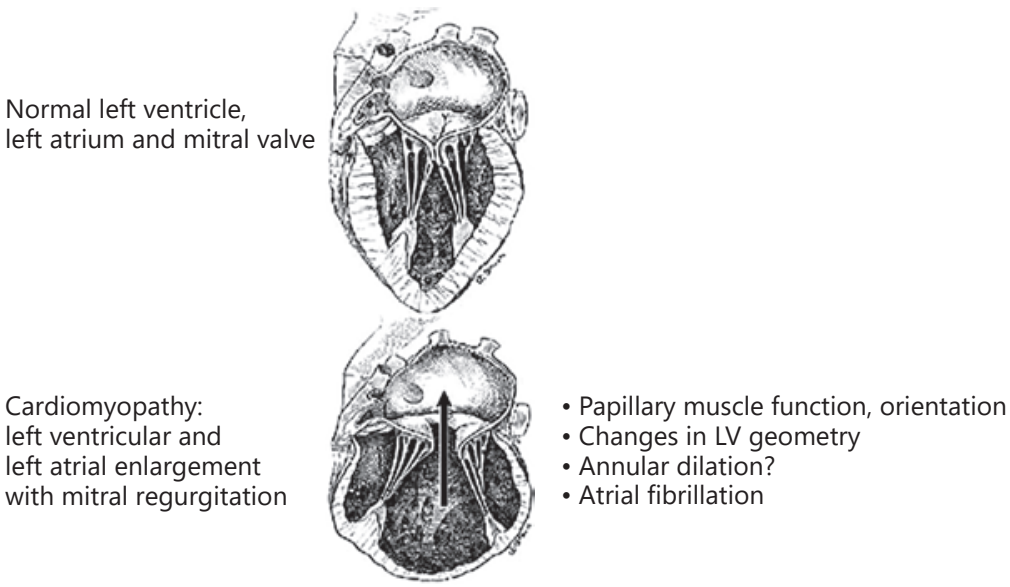

and dysfunction (Fig. 1) [1,2]; however, "MR begets MR," as it contributes to LV dilatation that further deteriorates MR leading to a vicious cycle. For this reason, secondary MR in the setting of heart failure and reduced ejection fraction is associated with higher rates of hospitalization and mortality $[3,4]$. Current guidelines recommend medical management and resynchronization therapy (when appropriate) for secondary MR as mitral valve surgery is associated with high risk of complications and does not provide a survival benefit [5-14]. More recently, observational and randomized controlled studies demonstrated the efficacy of transcatheter mitral valve repair in heart failure patients with significant MR [15-18]. It was hypothesized that eliminating the vicious cycle of MR in these patients will improve outcomes. Therefore, a meta-analysis of published studies was performed in order to evaluate the role of transcatheter mitral valve repair using the MitraClip device in heart failure patients with significant secondary MR, specifically examining the impact on mortality and rehospitalizations.

\section{Methods}

\section{Study Design}

Meta-analysis was conducted as per the PRISMA statement [19]. The methodological quality of the studies was assessed using the Downs and Black checklist [20].

\section{Data Sources}

PubMed, Cochran CENTRAL, and Embase databases were reviewed. The following terms "percutaneous mitral valve repair" or "transcatheter mitral valve repair" and "heart failure" were used for the literature search. Studies comparing medical therapy plus transcatheter mitral valve repair using MitraClip to medical therapy alone in heart failure patients with significant secondary MR were chosen. Furthermore, reference lists from relevant articles were reviewed to identify additional studies. Studies in languages other than English were excluded. In addition, case reports, editorials, case series, review articles, and studies with $<10$ patients were also excluded.

\section{Study Selection}

Clinical trials were included in the meta-analysis if the following criteria were met: (i) studies reported 1 of the following clinical outcomes in both treatment arms - (a) all-cause mortality; (b) rehospitalization; and/or (c) cardiovascular mortality; (ii) minimum follow-up period of at least 12 months; and (iii) studies involving patients with secondary MR.

\section{Data Extraction}

The titles and abstracts were screened for relevance by 2 of the authors (AV and $\mathrm{AH}$ ) independently; if differences were found, they were discussed until a consensus was reached. The manuscripts of selected titles/abstracts were assessed for inclusion, and the authors were contacted if further information was required. Using the selection criteria enlisted above, the 2 reviewers independently identified the papers to be included and excluded, and data from the included papers were extracted using predefined extraction flow sheets. Any differences in data extraction were discussed until agreement between both reviewers was reached. Device-related complications were defined as single leaflet detachment, device embolization, device implantation failure, cardiac tamponade, or urgent conversion to surgery. Periprocedural complications included death, stoke, myocardial infarction, significant bleeding, vascular injury, unplanned surgery, as well as device-related complications. In addition, mortality and rehospitalization data were obtained.

Data Analysis

Statistical analysis was performed with Review Manager (RevMan) software, version 5.3 (Copenhagen: The Nordic Cochrane Centre, The Cochrane Collaboration, 2012). A random-effects 
Fig. 2. PRISMA flow sheet showing studies

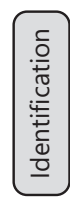

Records identified through
database searching $(n=602)$

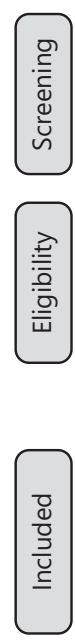

Additional records identified through other sources $(n=3)$

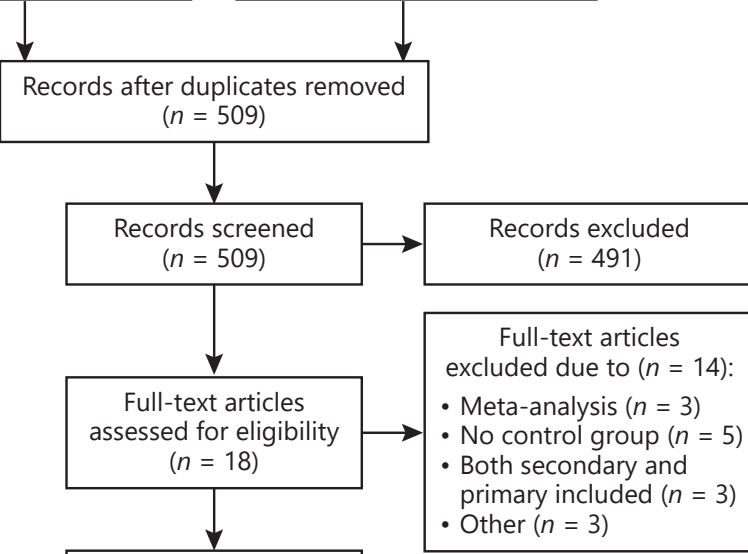

Studies included in qualitative synthesis $(n=4)$

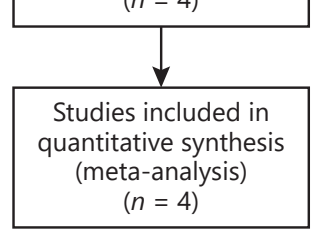
selected for analysis.

Table 1. Clinical characteristics of the included studies

\begin{tabular}{|c|c|c|c|c|c|c|c|c|c|c|c|c|c|c|}
\hline Author & \multicolumn{2}{|c|}{ Subjects, $n$} & \multicolumn{2}{|c|}{ Age, years } & \multicolumn{2}{|c|}{ Males, \% } & \multicolumn{2}{|c|}{ LVEF, \% } & \multicolumn{2}{|c|}{ NYHA $3-4, \%$} & \multicolumn{2}{|l|}{ ICM, \% } & \multicolumn{2}{|l|}{$\mathrm{AF}, \%$} \\
\hline Giannini et al. [23] & 232 & 151 & 71 & 71 & 73 & 74 & 34 & 32 & NR & NR & 45 & 50 & 33 & 33 \\
\hline Obadia et al. [17] & 152 & 152 & 70 & 71 & 79 & 70 & 33 & 33 & 63 & 70 & 63 & 56 & 35 & 33 \\
\hline Stone et al. [18] & 302 & 312 & 72 & 73 & 67 & 62 & 31 & 31 & 57 & 65 & 52 & 51 & 57 & 53 \\
\hline
\end{tabular}

Device indicates transcatheter mitral valve repair using the MitraClip device. AF, atrial fibrillation; LVEF, left ventricular ejection fraction; ICM, ischemic cardiomyopathy; MT, medical therapy; NR, not reported; NYHA, New York Heart Association functional class.

model with the Mantel-Haenszel method was used to calculate pooled odds ratio and 95\% confidence interval (CI) [21]. Three outcomes were assessed: (i) all-cause mortality; (ii) cardiovascular mortality; and (iii) rehospitalization. If data pertaining to hospitalization secondary to heart failure or cardiovascular causes were not presented in a study, the number of hospitalizations irrespective of etiology was used for analysis. The rates of device-related and periprocedural complications in the intervention arm were also analyzed. Heterogeneity between studies was assessed using Cochran's Q test and $I^{2}$ statistic, which denotes the percentage of total variation across studies that are a result of heterogeneity rather than chance. $I^{2}$ values of $<40 \%, 40-60 \%$, or $>60 \%$ indicated low, moderate, or substantial heterogeneity, respectively [22]. Publication bias was assessed by Begg's test and Egger's regression test.

\section{Results}

\section{Study Outlines and Characteristics}

Four studies [15-18] (Fig. 2), 2 randomized controlled (Obadia et al. [17], and Stone et al. [18]) and 2 nonrandomized studies (Armeni et al. [16], and Giannini et al. [15]), compared medical therapy plus transcatheter mitral valve repair using MitraClip to medical therapy alone in heart failure patients with moderate to severe secondary MR. Pooled analysis included a total of 1,421 heart failure patients, of which 746 patients underwent transcatheter mitral valve repair and 675 patients received 


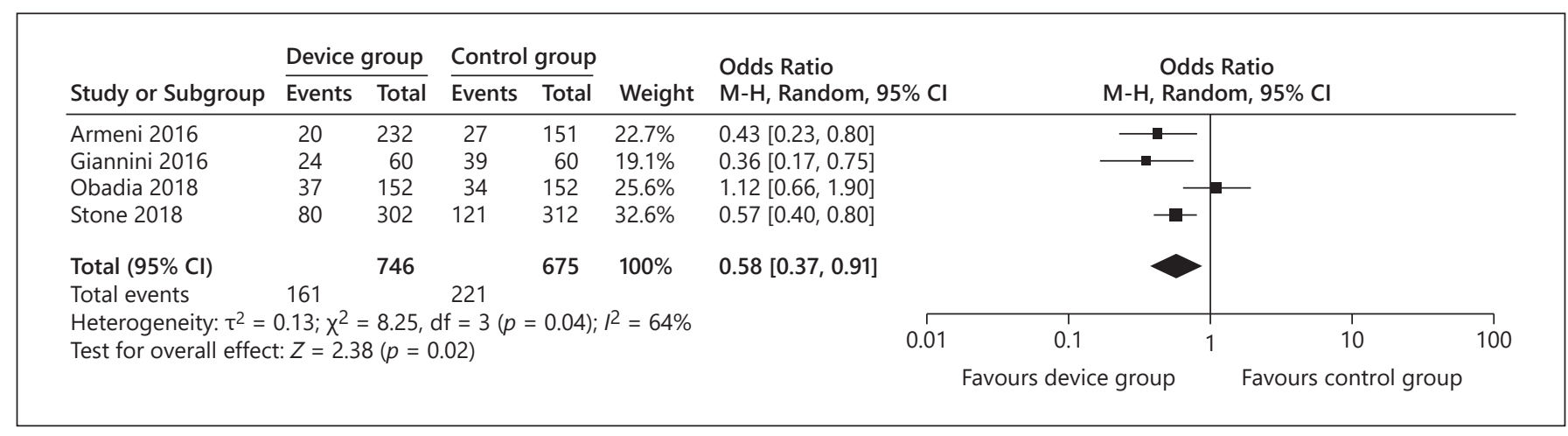

Fig. 3. Forest plot of risk of all-cause mortality. Pooled analysis of the 4 studies included 2 observational studies (Armeni et al. [16], and Giannini et al. [23]) and 2 randomized studies (Obadia et al. [17], and Stone et al. [18]). Meta-analysis of pooled studies revealed that in heart failure patients with secondary MR, medical therapy plus transcatheter mitral valve repair using the MitraClip device significantly reduced all-cause mortality compared with medical therapy alone (control). MR, mitral regurgitation; CI, confidence interval.

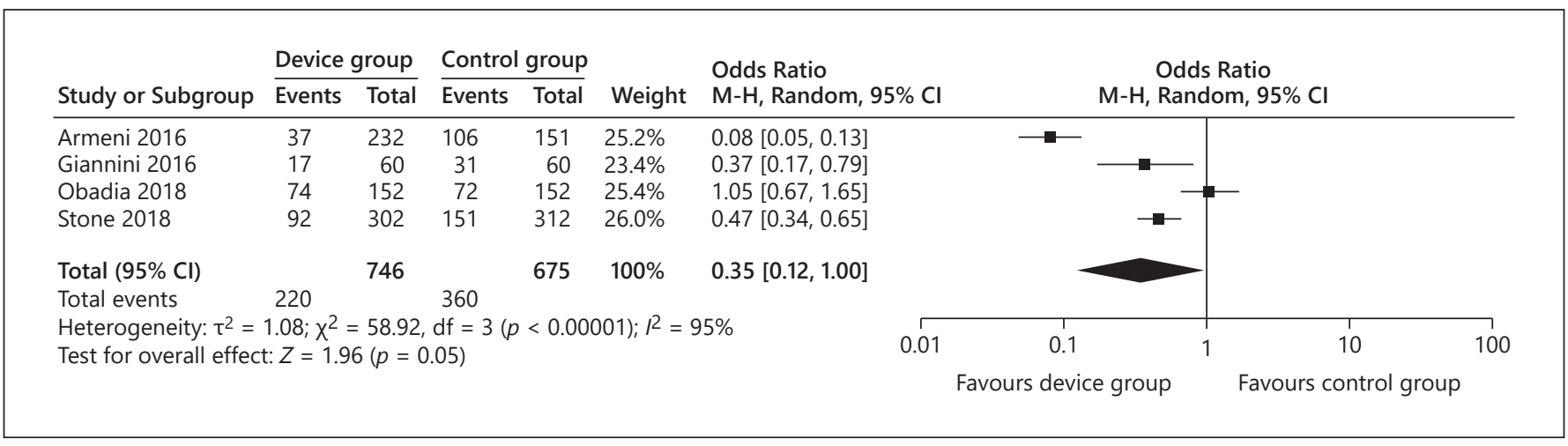

Fig. 4. Forest plot of risk of rehospitalizations. Meta-analysis of the 4 studies including 2 observational studies (Armeni et al. [16], and Giannini et al. [23]) and 2 randomized studies (Obadia et al. [17], and Stone et al. [18]). Meta-analysis of pooled studies revealed a trend toward lower rates of rehospitalizations in heart failure patients with secondary MR treated with medical therapy plus transcatheter mitral valve repair using the MitraClip device compared

medical therapy alone. Details of the studies are reported in Table 1. The duration of follow-up in the included studies ranged from 12 to 22.7 months.

\section{All-Cause Mortality}

Pooled analysis of the 4 studies reporting mortality in heart failure patients with secondary MR revealed that transcatheter mitral valve repair significantly reduced allcause mortality (OR 0.58 , 95\% CI $0.37-0.91 ; p=0.02$ ) (Fig. 3). There was substantial heterogeneity across the studies $\left(p=0.04, I^{2}=64 \%\right)$. The robustness of these findings was explored by changing the meta-analysis method from with medical therapy alone (control). It should be mentioned that this borderline significance related to rehospitalization, at least partially, is due to the fact that the mortality rate in patients who did not undergo MitraClip was higher; obviously a higher mortality resulted in a decreased rate of rehospitalization. MR, mitral regurgitation; $\mathrm{CI}$, confidence interval.

a random- to fixed-effects model; there was no change in summary effect size with a fixed-effects model (OR 0.60, 95\% CI 0.48-0.77; $p<0.0001)$. The 30 -day all-cause mortality in the intervention arm ranged from 0 to $2.3 \%$.

\section{Cardiovascular Mortality}

Three studies reported the effect of transcatheter mitral valve repair on cardiovascular mortality. Two studies revealed significant reduction in cardiovascular mortality with transcatheter mitral valve repair $[15,18]$. Conversely, the study by Obadia et al. [17], did not demonstrate a benefit in mortality [17]. 


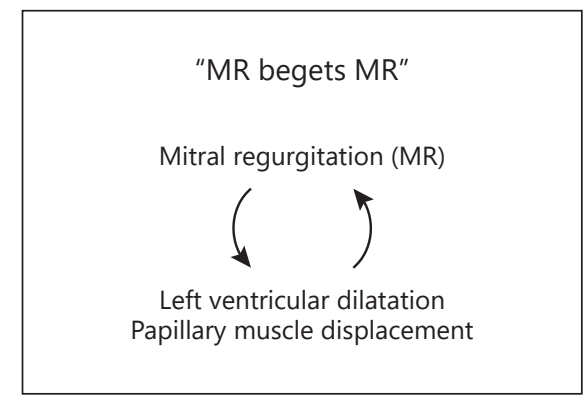

Fig. 5. Chronic mitral regurgitation (MR) can result in left ventricular dilatation with papillary muscle displacement, which in turn precipitates the severity of MR leading to a vicious cycle.

Device-Related and Periprocedural Complications

Device-related complications ranged from 1.3 to $7 \%$. Device implantation failure and single leaflet detachment occurred in 2.4 and $1.7 \%$, respectively. Periprocedural complications ranged from 7.5 to $14.6 \%$.

\section{Rehospitalization}

Four studies reported the impact of transcatheter mitral valve repair on the rate of rehospitalizations. There was a trend toward lower rates of rehospitalizations (OR $0.35,95 \%$ CI $0.12-1.00 ; p=0.05$ ) (Fig. 4). Substantial heterogeneity was noted across the studies $\left(p<0.001, I^{2}=\right.$ $95 \%)$. Switching to a fixed-effects model demonstrated benefit with transcatheter mitral valve repair (OR 0.39 , $95 \%$ CI $0.31-0.48 ; p<0.001)$. It should be mentioned that this borderline significance related to rehospitalization, at least partially, is due to the fact that the mortality rate in patients who did not undergo MitraClip was higher; obviously a higher mortality resulted in a decreased rate of rehospitalization.

\section{Discussion}

This meta-analysis demonstrated that medical therapy plus transcatheter mitral valve repair using MitraClip in patients with heart failure and secondary MR provides survival benefit compared to guideline-directed medical therapy alone. Furthermore, transcatheter mitral valve repair in this patient population may decrease the rates of rehospitalization. It should be mentioned that patients who survived due to undergoing the MitraClip procedure (i.e., patients who would have died without the procedure) contributed to the rate of hospitalizations. Trans- catheter mitral valve repair is associated with a reasonably low complication rate.

A prior meta-analysis reported a survival benefit with transcatheter mitral valve repair among heart failure patients with MR; however, the analysis included patients with secondary and organic (primary) MR [23]. Another meta-analysis demonstrated that transcatheter mitral valve repair with MitraClip in patients with heart failure and secondary MR decreased the severity of MR and improved functional capacity [24]; however, this study compared outcomes before and after mitral valve repair in the same cohort of patients and did not compare to a medical therapy alone arm [24]. To our knowledge, this is the first meta-analysis comparing transcatheter mitral valve repair plus medical therapy to medical therapy alone in heart failure patients with exclusively secondary MR.

Irrespective of severity of LV systolic dysfunction, MR is associated with worse outcomes [25]. However, it is not clear whether MR per se contributes to mortality or MR reflects a more severe disease [4]. As it was stated previously, "MR begets MR" in which MR contributes to LV dilatation leading to worsening $\mathrm{MR}$ resulting in a vicious cycle (Fig. 5). Elimination of the MR, therefore, breaks this vicious cycle and may improve outcomes. Prohibitive perioperative risk due to age and/or other comorbidities precludes mitral valve surgery in some patients [26]. Furthermore, surgical treatment of significant secondary MR in heart failure patients does not provide any survival benefit. Current guidelines give mitral valve surgery in patients with significant secondary MR a Class IIb (Level of Evidence: B) indication [13]. In the EVEREST II (Endovascular Valve Edge-to-Edge Repair) study, transcatheter mitral valve repair in patients deemed to be high surgical risk (surgical mortality risk $\geq 12 \%$ ) was associated with a 30 -day mortality rate of $4.8 \%$ [27]. Safety profile has improved further with an increase in operator experience. The 30-day all-cause mortality was as low as $2.3 \%$ in COAPT (Cardiovascular Outcome Assessment of the MitraClip Percutaneous Therapy) trial [18]. Transcatheter mitral valve repair, which has an overall lower procedural risk compared with surgery, is a reasonable alternative to surgery in patients with heart failure and symptomatic secondary MR.

This meta-analysis demonstrated improvement in survival and a trend toward reduction in rehospitalizations with transcatheter mitral valve repair. There was a divergence in the results reported in the 2 recently published randomized controlled studies, COAPT and MITRA-FR (Percutaneous Repair with the MitraClip Device for Severe Functional/Secondary Mitral Regurgitation).
Vallakati/Hasan/Boudoulas 
However, there are a number of reasons that may explain the difference in outcomes. First, the LV end-diastolic volume was greater in the MITRA-FR trial (LV EDVI 135 $\mathrm{mL} / \mathrm{m}^{2}$ vs. $101 \mathrm{~mL} / \mathrm{m}^{2}$ ) likely reflecting more advanced stage of heart failure. Second, the mean effective regurgitant orifice area was larger in the COAPT trial (41 vs. 31 $\mathrm{mm}^{2}$ ) suggesting patients included in this trial had more severe MR. Third, enrollment in the COAPT trial was designed to include patients who were truly refractory to medical therapy. When compared with the MITRA-FR trial, patients in the medical therapy arm of COAPT trial had higher baseline natriuretic peptide levels and were hospitalized more frequently for heart failure [17, 18]. Last, when compared with the COAPT trial, a higher proportion of patients in the MITRA-FR trial had severe residual MR at 12-month follow-up suggesting differences in procedural performance $[17,18]$. Another randomized trial, RESHAPE-HF2 (A RandomizEd Study of tHe MitrACliP DEvice in Heart Failure Patients With Clinically Significant Functional MR; NCT02444338) is currently underway and will provide additional information on the role of transcatheter mitral valve repair in patients with heart failure [28].

\section{Limitations}

Our pooled analysis has several limitations. Due to the small sample size of relevant studies, data from randomized controlled trials and nonrandomized studies were included. Inclusion of observational studies is associated with a potential bias, as the distribution of unmeasured confounding factors may not be similar between 2 com- parison groups. Systematic reviews may suffer from publication bias due to probability of publication of only positive studies or omission of pertinent studies from analysis [29]. However, comprehensive literature search of multiple databases was performed and publication bias was evaluated by Begg's test and Egger's regression test.

\section{Conclusions}

MR may contribute to LV dilatation, which in turn increases the severity of MR leading to a vicious cycle; eliminating the vicious cycle may improve outcomes. Evidence from pooled analysis suggests that transcatheter mitral valve repair using MitraClip on top of medical therapy, in appropriately selected symptomatic heart failure patients with significant secondary MR, provides survival benefit and may decrease hospitalizations when compared to guideline-directed medical therapy alone. Transcatheter mitral valve repair, therefore, should be considered in these patients. Sound clinical judgment and common sense should be applied to identify individual patients who most likely will benefit from this procedure.

\section{Conflict of Interest Statement}

All authors have no conflict of interest relevant to the topic in discussion.

\section{Funding Sources}

The authors did not receive any funding.

\section{References}

1 Boudoulas KD, Borer JS, Boudoulas H. Etiology of valvular heart disease in the 21st century. Cardiology. 2013;126(3):139-52.

2 Asgar AW, Mack MJ, Stone GW. Secondary mitral regurgitation in heart failure: pathophysiology, prognosis, and therapeutic considerations. J Am Coll Cardiol. 2015;65(12): 1231-48.

3 Goliasch G, Bartko PE, Pavo N, Neuhold S, Wurm R, Mascherbauer J, et al. Refining the prognostic impact of functional mitral regurgitation in chronic heart failure. Eur Heart J. 2018;39(1):39-46.

4 Sannino A, Smith RL 2nd, Schiattarella GG, Trimarco B, Esposito G, Grayburn PA. Survival and cardiovascular outcomes of patients with secondary mitral regurgitation: a sys- tematic review and meta-analysis. JAMA Cardiol. 2017;2(10):1130-9.

5 Nishimura RA, Otto CM, Bonow RO, Carabello BA, Erwin JP, Guyton RA, et al. 2014 AHA/ACC guideline for the management of patients with valvular heart disease: executive summary: a report of the american college of cardiology/american heart association task force on practice guidelines. J Am Coll Cardiol. 2014;63(22):2438-88.

6 Nijst P, Martens P, Mullens W. Heart failure with myocardial recovery: the patient whose heart failure has improved: what next? Prog Cardiovasc Dis. 2017;60(2):226-36.

7 Eriksson SV, Eneroth P, Kjekshus J, Offstad J, Swedberg K. Neuroendocrine activation in relation to left ventricular function in chronic severe congestive heart failure: a subgroup analysis from the cooperative north scandinavian enalapril survival study (CONSENSUS). Clin Cardiol. 1994;17(11):603-6.

8 Granger CB, McMurray JJ, Yusuf S, Held P, Michelson EL, Olofsson B, et al. Effects of candesartan in patients with chronic heart failure and reduced left-ventricular systolic function intolerant to angiotensin-converting-enzyme inhibitors: the CHARMalternative trial. Lancet. 2003;362(9386): $772-6$.

9 Krum H, Roecker EB, Mohacsi P, Rouleau JL, Tendera M, Coats AJ, et al. Effects of initiating carvedilol in patients with severe chronic heart failure: results from the COPERNICUS study. JAMA. 2003;289(6):712-8.
Meta-Analysis of Transcatheter Mitral Valve in Heart Failure 
10 Pitt B, Zannad F, Remme WJ, Cody R, Castaigne A, Perez A, et al. The effect of spironolactone on morbidity and mortality in patients with severe heart failure. Randomized aldactone evaluation study investigators. N Engl J Med. 1999;341(10):709-17.

11 Yusuf S, Yusuf S, Pitt B, Davis CE Jr, Hood WB, Cohn JN. Effect of enalapril on mortality and the development of heart failure in asymptomatic patients with reduced left ventricular ejection fractions. N Engl J Med. 1992; 327(10):685-91.

12 Kang DH, Park SJ, Shin SH, Hong GR, Lee S, Kim MS, et al. Angiotensin receptor neprilysin inhibitor for functional mitral regurgitation. Circulation. 2019;139(11):1354-65.

13 Nishimura RA, Otto CM, Bonow RO, Carabello BA, Erwin JP, Fleisher LA, et al. 2017 AHA/ACC focused update of the 2014 AHA/ ACC guideline for the management of patients with valvular heart disease: a report of the American College of Cardiology/American Heart Association task force on clinical practice guidelines. Circulation. 2017; 135(25):e1159-89.

14 Baumgartner H, Falk V, Bax JJ, De Bonis M, Hamm C, Holm PJ, et al. 2017 ESC/EACTS guidelines for the management of valvular heart disease. Eur Heart J. 2017;38(36):2739_ 91.

15 Giannini C, Fiorelli F, De Carlo M, Guarracino F, Faggioni M, Giordano P, et al. Comparison of percutaneous mitral valve repair versus conservative treatment in severe functional mitral regurgitation. Am J Cardiol. 2016; 117(2):271-7.
16 Armeni P, Boscolo PR, Tarricone R, Capodanno D, Maggioni AP, Grasso C, et al. Realworld cost effectiveness of mitraclip combined with medical therapy versus medical therapy alone in patients with moderate or severe mitral regurgitation. Int J Cardiol. 2016;209:153-60.

17 Obadia JF, Messika-Zeitoun D, Leurent G, Iung B, Bonnet G, Piriou N, et al. Percutaneous repair or medical treatment for secondary mitral regurgitation. N Engl J Med. 2018; 379(24):2297-306.

18 Stone GW, Lindenfeld J, Abraham WT, Kar S, Lim DS, Mishell JM, et al. Transcatheter mitral-valve repair in patients with heart failure. N Engl J Med. 2018;379(24):2307-18.

19 Moher D, Liberati A, Tetzlaff J, Altman DG. Preferred reporting items for systematic reviews and meta-analyses: the PRISMA statement. BMJ. 2009;339:b2535.

20 Downs SH, Black N. The feasibility of creating a checklist for the assessment of the methodological quality both of randomised and nonrandomised studies of health care interventions. J Epidemiol Community Health. 1998; 52(6):377-84

21 DerSimonian R, Laird N. Meta-analysis in clinical trials revisited. Contemp Clin Trials. 2015;45(Pt A):139-45

22 Higgins JP, Thompson SG, Deeks JJ, Altman DG. Measuring inconsistency in meta-analyses. BMJ. 2003;327(7414):557-60.
23 Giannini C, D’Ascenzo F, Fiorelli F, Spontoni P, Swaans MJ, Velazquez EJ, et al. A metaanalysis of mitraclip combined with medical therapy vs. medical therapy alone for treatment of mitral regurgitation in heart failure patients. ESC Heart Failure. 2018;5(6):11508.

24 De Rosa R, Silverio A, Baldi C, Di Maio M, Prota C, Radano I, et al. Transcatheter repair of functional mitral regurgitation in heart failure patients: a meta-analysis of 23 studies on mitraclip implantation. Circ J. 2018; 82(11):2800-10.

25 Grigioni F, Enriquez-Sarano M, Zehr KJ, Bailey KR, Tajik AJ. Ischemic mitral regurgitation: long-term outcome and prognostic implications with quantitative doppler assessment. Circulation. 2001;103(13):1759-64.

26 Mirabel M, Iung B, Baron G, Messika-Zeitoun D, Détaint D, Vanoverschelde JL, et al. What are the characteristics of patients with severe, symptomatic, mitral regurgitation who are denied surgery? Eur Heart J. 2007;28(11): 1358-65.

27 Glower DD, Kar S, Trento A, Lim DS, Bajwa T, Quesada R, et al. Percutaneous mitral valve repair for mitral regurgitation in high-risk patients: results of the EVEREST II study. J Am Coll Cardiol. 2014;64(2):172-81.

28 RESHAPE-HF2 (A RandomizEd Study of tHe MitrACliP DEvice in Heart Failure Patients With Clinically Significant Functional Mitral Regurgitation). Available from: https://clinicaltrials.gov/ct2/show/NCT02444338.

29 Boudoulas KD, Leier CV, Geleris P, Boudoulas $\mathrm{H}$. The shortcomings of clinical practice guidelines. Cardiology. 2015;130(3):187-200. 